\title{
Analysis of the Relationship Between Pineal Hormone Melatonin Level and Occupational Mercury Exposure in Ex-miners with Machine Learning Methods
}

\author{
Joško Osredkar, Bernard Ženko,2, Darja Kobal Grum,3, Mladen Krsnik, \\ Sašo Džeroski, Milena Horvat, ${ }^{6}$ and Alfred B. Kobal ${ }^{7}$
}

\begin{abstract}
The aim of this study was an analysis of the relationship between pineal hormone melatonin level and long-term occupational exposure to elemental mercury vapour $\left(\mathrm{Hg}^{\circ}\right)$ of ex-mercury miners. Melatonin (MEL) is a hormonal product of the pineal gland. $\mathrm{Hg}$ accumulation in the pineal gland in ex-miners could modify the synthesis of melatonin and there are no data available in the scientific literature on the possible effects of $\mathrm{Hg}$ on melatonin excretion.

Regression/model trees were used to perform the analyses and investigate the interactions between melatonin secretion and excretion on one hand, and various indicators of occupational $\mathrm{Hg}$ exposure and some other medical variables on the other. The results point us towards some previously unknown factors that influence the excretion of melatonin and support some assumptions that melatonin also has antioxidative effect. In the process of antioxidation activity melatonin decomposes and we assume this is the reason for decreased excretion of melatonin in mercury miners.
\end{abstract}

\section{Introduction}

The toxic effects of mercury are well known and in the case of occupational exposure to $\mathrm{Hg}^{\circ}$ the most frequent symptoms and signs include erythrism, increased irritability, depression, insomnia, psychotic disturbances, tremor and renal impairment (WHO, 1976, 1991). High $\mathrm{Hg}$ and Se retention and co-accumulation (near 1:1 molar ratio) have been found in the brain, endocrine glands and also pineal gland in ex-miners from the Idrija

\footnotetext{
${ }^{1}$ Institute of Clinical Chemistry and Biochemistry, University Medical Centre, Slovenia

${ }^{2}$ Department of Knowledge Technologies, Jožef Stefan Institute, Jamova 39, SI-1000 Ljubljana, Slovenia; bernard.zenko@ijs.si

${ }^{3}$ Department of Psychology, University of Ljubljana, Slovenia

${ }^{4}$ Institute of Clinical Chemistry and Biochemistry, University Medical Centre, Slovenia

${ }^{5}$ Department of Knowledge Technologies, Jožef Stefan Institute, Slovenia

${ }^{6}$ Department of Environmental Sciences, Jožef Stefan Institute, Slovenia

${ }^{7}$ Idrija Mercury Mine, Slovenia
} 
Mercury Mine even several years after exposure (Kosta et al., 1975; Falnoga et al., 2000). In our recent study (Kobal et al., 2004) elevated melatonin (MEL) level in blood of exmercury miners was found. $\mathrm{Hg}$ accumulation in the pineal gland in retired miners could modify the synthesis of melatonin and there are no data available in the scientific literature on the possible effects of $\mathrm{Hg}$ on melatonin excretion. Melatonin, N-acetyl-5methoxytryptamine, is a hormonal product of the pineal gland. Its synthesis is higher at night than during the day. Once melatonin is produced in the pineal gland it is quickly released into the vascular system. The interactions of melatonin membrane-bound receptors are believed to mediate the endocrine and circadian rhythm effects of melatonin. Recently, the pineal hormone melatonin was found to have a potent-free-radical scavenging activity and the protective effect of melatonin towards peroxidative damage was found in some in vivo and in vitro studies (Reiter et al., 1995). Hypothetically, this melatonin activity could play a key role in interaction with known $\mathrm{Hg}$ free radical generation (Jansson and Harms-Ringdahl, 1993; Lund et al., 1993).

In studies (Kobal et al., 2004; Kobal-Grum et al., 2004), no association between melatonin levels in blood and urine, and observed external and biological indices of past occupational exposure were found. The aim of the present study was to investigate the possible associations between melatonin levels in blood and urine, and indicators of miners past occupational $\mathrm{Hg}^{\circ}$ exposure in combination with some additional variables.

The rest of the paper is organized as follows. The next section describes the study population, the data collected, and the data analysis methodology. Section 3 gives the results of this study, while in the last section discussion and conclusions are presented.

\section{Material and methods}

\subsection{Study population}

Initially, 120 males were examined in the study. After the selection procedure, the study population comprised 53 mercury miners and 53 workers from the control group. The mean age of miners was 45 (range 29-62), of controls 43 (range 30-62) years. The study group of miners comprised 33 active miners not exposed to $\mathrm{Hg}^{\circ}$ in the previous 8 to 60 months, and 20 retired miners who prior to the present observation were not exposed to $\mathrm{Hg}^{\circ}$ from 32 to 336 months. In their work miners had been intermittently exposed to $\mathrm{Hg}^{\circ}$. The $\mathrm{Hg}^{\circ}$ concentrations in the air varied, depending on specific workplaces, from 0.05 to over $1.00 \mathrm{mg} / \mathrm{m}^{3}$. During exposure to $\mathrm{Hg}^{\circ}$, all miners used personal protective equipment, i.e., half masks or Racal helmets with $\mathrm{Hg}^{\circ}$ absorbing filters. No individuals in the study were occupationally exposed to cadmium or lead.

\subsection{Medical examination}

The medical examination included a medical history, some behavioral and biological risk factors for cardiovascular disease (CVD) (Leparski and Nüssel, 1987) and an evaluation of clinical neurological status. A dental amalgam score was calculated with the methodology proposed by (Aposhian et al., 1992). The examination also included blood and urine sampling for determination of: 1) total blood mercury (B-THg) and urine mercury (U- 
$\mathrm{Hg}$ ), 2) pineal hormone melatonin in blood (B-MEL) and urine (U-MEL), 3) Se levels in blood, plasma and urine, 4) CuZn-SOD, CAT, GSH-Px activity in erythrocytes as indices of free radical activity, 5) malondialdehyde (MDA), a lipid peroxidation product, and 6) lipid profile and other basic laboratory analyses including cadmium $(\mathrm{Cd})$ and lead $(\mathrm{Pb})$ in blood. Psychological examination was also carried out (Kobal-Grum et al., 2004).

\subsection{Assessment of occupational $\mathrm{Hg}^{\circ}$ exposure}

Environmental and biological data on the group of miners studied were collected from 1959 to 2000 from workload records, daily reports on $\mathrm{Hg}^{\circ}$ measurements in the workplace and personal medical records and biological monitoring data. On the basis of collected data, the external exposure indices and biological exposure indices (internal dose) were evaluated. The following environmental indices of $\mathrm{Hg}^{\circ}$ were calculated for each miner: 1) years of work in the mercury mine (years of exposure), 2) cycles of exposure (intervals of work exposed to $\mathrm{Hg}^{\circ}$ ), 3) average time-weighted (ATW) air $\mathrm{Hg}^{\circ}$ concentration in $\mathrm{mgHg} / \mathrm{m}^{3}$ air, and 4) integrated exposure intensity (IEI) of the whole exposure period expressed in $\mathrm{mgHg} / \mathrm{m}^{3}$ hours. Since 1959 , the miners were biologically monitored by means of urine mercury (U-Hg) analyses. Biological exposure indices were evaluated on the basis of $5452 \mathrm{U}-\mathrm{Hg}$ measurements performed during the cycles of miners' exposure to $\mathrm{Hg}^{\circ}$. Based on these data, the following biological indices of occupational exposure were calculated: 1) geometric mean of cycles U-Hg level, calculated from all urine samples determined during the cycles of exposure expressed in $\mu \mathrm{gHg} / \mathrm{L}$, and 2) geometric mean of cycles peak U-Hg level, calculated from all cycles peak U-Hg levels determined during the cycles of exposure expressed in $\mu \mathrm{gHg} / \mathrm{L}$. The actual background exposure to inorganic and methyl mercury was evaluated by determination of B-THg and U-Hg. Background exposure to $\mathrm{Cd}$ and $\mathrm{Pb}$ was also evaluated.

\subsection{Blood and urine sampling}

Venous peripheral blood samples were collected between 6:30 and 7:30 a.m. in $7 \mathrm{~mL}$ sterile Vacutainer tubes containing lithium heparin as an anticoagulant. An aliquot for melatonin determination was taken and stored at $-40^{\circ} \mathrm{C}$ until the analysis. The rest of the blood was centrifuged at $1000 \mathrm{~g}$ for 15 minutes at $4^{\circ} \mathrm{C}$ and plasma was separated. Erythrocytes were washed four times with isotonic saline. Centrifugation for each wash was carried out at $1000 \mathrm{~g}$ and $4^{\circ} \mathrm{C}$ for 10 minutes. Washed erythrocytes were stored at $-40^{\circ} \mathrm{C}$. 8 hour urine (night-morning urine) was collected in metal-free polypropylene tubes during the night (22 p.m. -6 a.m.). Blood and urine samples were brought to the laboratory within 1 hour after collection at $4^{\circ} \mathrm{C}$.

\subsection{Biological analyses}

Blood and urine MEL was determined by the ELISA (Enzyme-Linked Immunosorbent Assay) method (IBL-Hamburg). The 6-sulphatoxymelatonine ELISA kit provides mate-

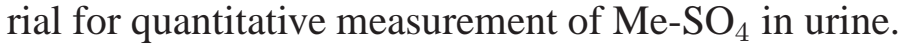

Total mercury in whole blood (B-THg) was determined by cold vapor atomic absorption spectrometry (CV AAS). Plasma and urine selenium (P-Se and $\mathrm{U}-\mathrm{Se}$ ), $\mathrm{Cd}$ and $\mathrm{Pb}$ in 
blood were determined by Zeeman graphite furnace atomic absorption spectrometry (GF AAS) using a palladium chemical modifier. Analyses of other biological variables are presented in previous publications (Kobal et al., 2004; Kobal-Grum et al., 2004).

\subsection{Data analysis methodology}

\subsubsection{Statistical analyses}

The group differences in all observed parameters were evaluated by applying an analysis of variance using one-way ANOVA software. The relationship between exposure and other variables was evaluated by means of Pearson's correlation coefficient, which reflects the degree of linear relation between two sets of data.

\subsubsection{Machine learning methods}

Machine learning methods were used in order to find possible explanations of associations between the target variables (concentrations of melatonin in urine and blood) and biological indicators of occupational $\mathrm{Hg}^{\circ}$ exposure in combination with other variables. More specifically, we used model trees (Quinlan, 1992), which are a generalization of regression trees (Breiman et al., 1984). Regression trees are a formalism for representation of piece-wise constant functions, while model trees are more general and are a formalism for representation of piece-wise linear functions. In the following, we only discuss model trees since regression trees are just a special case. Like classical regression equations, model trees predict the value of a dependent variable (called a class) from the values of a set of independent variables (called attributes). Data represented in the form of a table can be used for learning or construction of a model tree. In the table, each row (example or subject) has the form $\left(x_{1}, x_{2}, \ldots, x_{N}, y\right)$, where $x_{i}$ are values of $N$ attributes (e.g., subjects' age, daily consumption of alcohol, etc.) and $y$ is the value of the class (e.g., the melatonin concentration in blood). Unlike classical regression approaches, which find a single equation for a given set of data, model trees partition the space of examples into axis-parallel rectangles and fit a model to each of these partitions. A model tree has a test in each inner node which tests the value of a certain attribute, and in each leaf a model for predicting the class: the model can be a linear equation or merely a constant. An example of a model tree can be seen in Figure 1. Given a new example (subject) for which the value of the class $(y)$ should be predicted, the tree is interpreted from the root $\left(x_{1}\right)$. In each inner node, the prescribed test is performed and according to the result, the corresponding left or right sub-tree is selected. When the selected node is a leaf, the value of the class for the new example is predicted according to the model in the leaf $\left(L M_{1}, L M_{2}\right.$, or $L M_{3}$ ).

\section{Model tree construction}

The first step in the construction of a model tree is the construction of the initial regression tree. Regression tree construction proceeds recursively, starting with the entire set of training examples in one node. At each step, the most discriminating attribute is selected as the root of the (sub)tree and the current training set is split into two subsets according to the values of the selected attribute. Selection of the most discriminating attribute and its 


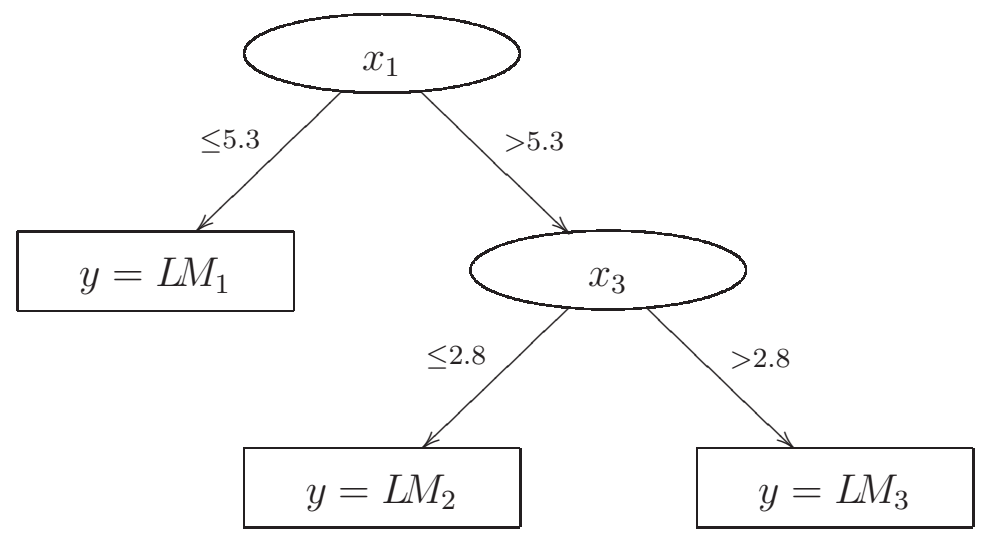

$$
\begin{aligned}
& L M_{1}=0.24 x_{2}+2.54 x_{3}+2.54 \\
& L M_{2}=4.04 x_{3}-0.24 \\
& L M_{3}=18.5
\end{aligned}
$$

Figure 1: An example of a model tree with three leaves $\left(L M_{1}\right.$ to $\left.L M_{3}\right)$.

split value is based on treating the standard deviation of class values of training examples at the current node as a measure of error at this node and maximizing the expected error reduction if that node is split in two nodes. Tree construction stops when the variance of class values of all examples in a node is small enough, or if only a small number of examples remain in the node. Such nodes are called leaves and are labeled with a constant value (mean class value of examples in a leaf) for predicting the class value.

The second step in model tree construction is calculation of linear models for each interior node (all nodes except leaves). Linear models are calculated using standard regression techniques, but only attributes tested in the subtree below the current node are used. The resulting linear model is simplified by dropping attributes one by one (greedy search) for as long as the error estimate decreases.

The next step is pruning of the tree, which reduces its size and improves accuracy. The tree is pruned back from the leaves. If the estimated error of a subtree is bigger than the estimated error of the linear model in the current node, the subtree is discarded and the previously internal node becomes a leaf with a linear model attached.

The last step in model tree construction is smoothing of the tree. Smoothing compensates for sharp discontinuities that occur between adjacent models at the leaves of the pruned tree. The idea is that the class value predicted by a leaf model is combined with the values predicted by models along the path to the root of the tree where the final prediction is computed. However, the same effect can be obtained by appropriately modifying linear models at the leaves. Additional details of model tree construction are concerned with dealing with non-numerical attributes and missing values; these can be found in (Wang et al., 1997) or (Witten and Frank, 1999).

\subsubsection{Machine learning experimental setup}

There are a number of systems for inducing regression and model trees, such as RETIS (Karalič, 1992) and M5 (Quinlan, 1992). The latter is one of the most well-known systems 
for regression and model tree induction. We used the M5' system (Wang and Witten, 1997), a re-implementation of M5 within the WEKA software package (Witten and Frank, 1999). The parameters of M5' were set to their default values.

A separate model tree for concentration of melatonin in blood (B-MEL) and in urine (U-MEL) was induced on the following features:

- group (active but not exposed to $\mathrm{Hg}^{\circ}$ ex-mercury miners - underground work, retired ex-mercury miners - underground work, and controls - work in the open),

- age,

- number of cigarettes per day,

- number of years smoking,

- number of years drinking alcohol,

- alcohol consumption in $\mathrm{ml} /$ day,

- total working time,

- body mass index (BMI),

- systolic and diastolic blood pressure,

- tremor frequency and character,

- albumin in urine $\mathrm{g} / \mathrm{mol}$ creatinine (a potential marker of the effect of $\mathrm{Hg}$ exposure),

- $\alpha_{1} \mu$ globulin in urine $\mathrm{g} / \mathrm{mol}$ creatinine,

- total $\mathrm{Hg}$ in plasma,

- Se-Hg ratio in plasma,

- Se in plasma (P-Se)in $\mu \mathrm{g} / \mathrm{L}$ and in urine (U-Se) in $\mu \mathrm{g} / \mathrm{g}$ creatinine,

- corrected $\mathrm{Hg}$ in urine to $1 \mathrm{~L}$,

- cadmium and lead in blood (B-Cd and B-Pb),

- SOD, catalase, and glutation peroxidase in erythrocytes,

- LDL and LP-a lipids in serum,

- LOOH, U-8OHdG, and MDA markers,

- integrated exposure intensity (IEI) score,

- number of work cycles of $\mathrm{Hg}$ exposure,

- average time-weighted (ATW) air $\mathrm{Hg}^{\circ}$ concentration,

- geometric mean of cycles U-Hg level in $\mu \mathrm{g} / \mathrm{L}$,

- the geometric mean of peak cycles $\mathrm{U}-\mathrm{Hg}$ level in $\mu \mathrm{g} / \mathrm{L}$,

- cumulative $\mathrm{U}-\mathrm{Hg}$ and cumulative peak $\mathrm{U}-\mathrm{Hg}$ levels in $\mu \mathrm{g} / \mathrm{L}$,

- time since last exposure in days (exposure-free interval), and

- presence of prenatal exposure to $\mathrm{Hg}$. 
Table 1: Indices of past occupational $\mathrm{Hg}^{\circ}$ exposure in miners.

\begin{tabular}{lrrr}
\hline & Mean & SD & Range \\
\hline Years of exposure & 14.9 & 5.5 & $7-31$ \\
Cycles of exposure & 41 & 21 & $13-119$ \\
ATWE $\left(\mathrm{mg} \mathrm{Hg}^{\circ} / \mathrm{m}^{3}\right)$ & 0.29 & 0.08 & $0.13-0.47$ \\
IEI $($ score $)$ & 1428 & 2108 & $105-10907$ \\
Cycles U-Hg level $(\mu \mathrm{g} / \mathrm{L})$ & 53.1 & 20.5 & $20-120$ \\
Cycles peak U-Hg level $(\mu \mathrm{g} / \mathrm{L})$ & 77.2 & 23.0 & $40-134$ \\
Cumulative U-Hg level $(\mu \mathrm{g} / \mathrm{L})$ & 6584 & 4444 & $1286-21390$ \\
Cumulative peak U-Hg level $(\mu \mathrm{g} / \mathrm{L})$ & 3900 & 2196 & $794-11365$ \\
\hline
\end{tabular}

Table 2: Characteristics of the observed groups.

\begin{tabular}{|c|c|c|c|c|c|}
\hline & \multicolumn{2}{|c|}{ Miners } & \multicolumn{2}{|c|}{ Controls } & \multirow[b]{2}{*}{$\mathrm{p}$-value } \\
\hline & Mear & $\mathrm{SD}$ & Mean & $\mathrm{SD}$ & \\
\hline Dental amalgam score & 12.8 & 12.4 & 12.5 & 10.9 & NS \\
\hline Fish meals per week & 0.52 & 0.96 & 0.59 & 0.88 & NS \\
\hline Cigarettes per day $^{a}$ & 21.6 & 7.3 & 20.5 & 9.5 & NS \\
\hline Alcohol $(\mathrm{ml} / \text { day })^{b}$ & 35.2 & 40.2 & 22.4 & 18.6 & NS \\
\hline Body mass index $\left(\mathrm{kg} / \mathrm{m}^{2}\right)$ & 27.8 & 4.1 & 27.4 & 4.1 & NS \\
\hline \multicolumn{6}{|l|}{ Blood pressure ${ }^{c}$} \\
\hline Systolic (mmHg) & 134. & 13.3 & 125.9 & 13.8 & $<0.01$ \\
\hline Diastolic (mmHg) & 87.9 & 8.8 & 81.2 & 11.1 & $<0.01$ \\
\hline U-albumin (g/mol creat.) & 1.36 & 1.78 & 0.85 & 0.44 & $<0.05$ \\
\hline $\mathrm{U}-\alpha_{1} \mu$ globulin (b/mol creat.) & $1.3 \mathrm{~s}$ & 2.67 & 0.61 & 0.35 & $<0.05$ \\
\hline \multicolumn{6}{|c|}{$\begin{array}{l}a \% \text { of smokers: miners } 59, \text { controls } 41 \\
b \% \text { of alcohol consumers over } 20 \mathrm{ml} / \text { day: miners } 28, \text { controls } 19, \text { Pearson's } \chi^{2} \text { NS } \\
{ }^{c} \text { hypertension over } 140 / 90 \mathrm{mmHg} \text { : miners } 22 \text {, controls } 12, \text { Pearson's } \chi^{2} \text { NS }\end{array}$} \\
\hline & Miners & & Controls & & \\
\hline & Mean & SD & Mean & $\mathrm{SD}$ & $\mathrm{p}$-value \\
\hline $\mathrm{B}-\mathrm{THg}(\mu \mathrm{g} / \mathrm{L})$ & 2.5 & 1.5 & 2.5 & 1.2 & NS \\
\hline $\mathrm{U}-\mathrm{Hg}(\mu \mathrm{g} / \mathrm{L})$ & 2.1 & 1.4 & 1.4 & 1.1 & $<0.01$ \\
\hline $\mathrm{P}-\mathrm{Se}(\mu \mathrm{g} / \mathrm{L})^{a}$ & 71.4 & 12.4 & 77.3 & 13.2 & $<0.05$ \\
\hline $\mathrm{U}-\mathrm{Se}(\mu \mathrm{g} / \mathrm{g}$ creat. $)$ & 16.5 & 6.6 & 14.0 & 6.9 & $<0.05$ \\
\hline B-MEL (ng/L) & 44.3 & 38.7 & 14.9 & 9.2 & $<0.01$ \\
\hline U-MEL sulphate $(\mu \mathrm{g} / \mathrm{L})$ & 31.8 & 40.7 & 46.9 & 43.2 & $<0.01$ \\
\hline U-MDA ( $\mu \mathrm{mol} / \mathrm{mmol}$ creat.) & 0.32 & 0.31 & 0.19 & 0.30 & $<0.01$ \\
\hline
\end{tabular}

For abbrevations see materials and methods section.

${ }^{a}$ Negative correlation with biological past $\mathrm{Hg}^{\circ}$ exposure indices: $r=-0.38, p<0.01$ 
Table 4: Linear regression model (model tree with a single leaf) constructed by M5', describing the concentration of melatonin in blood (B-MEL); correlation coefficient $=0.57$. The numbers in brackets (if present) correspond to the minimal, maximal, average, median values, and the relative importance factor of each numerical attribute. Relative importance factor is the product of an average value and a corresponding coefficient in the model. For nominal attributes, only number of subjects for which the condition is fulfilled and the number of all subjects are given.

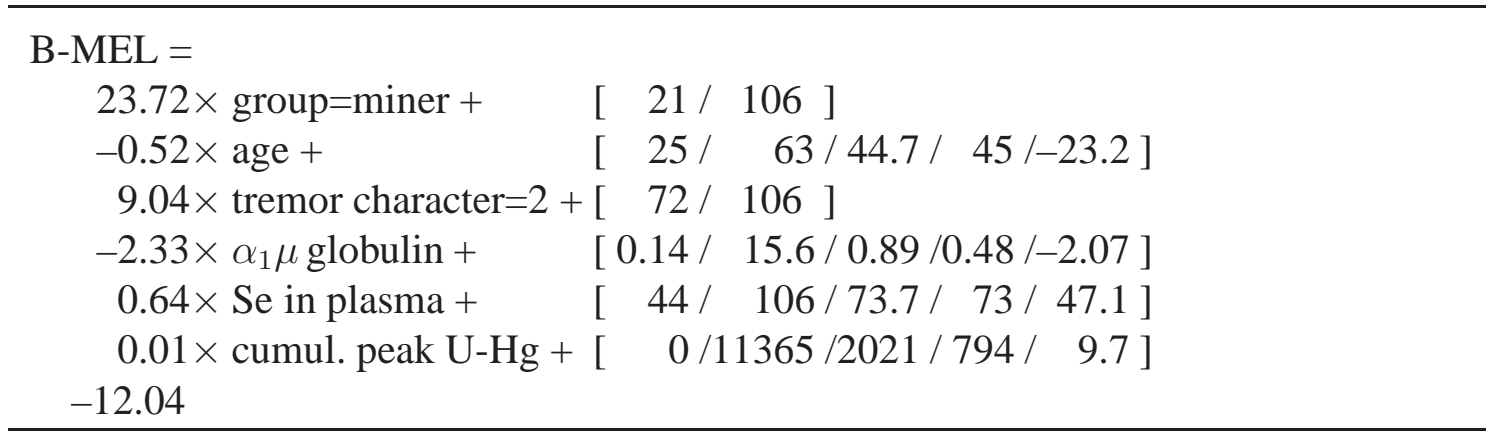

\section{Results}

\subsection{Statistical analysis results}

The indices of past occupational exposure to $\mathrm{Hg}^{\circ}$ are presented in Table 1. The mercury miners were intermittently exposed to $\mathrm{Hg}^{\circ}$ for periods of 7 to 31 years. The total number of exposure periods varied from 13 to 119 . The cumulative U-Hg peak level varied from 794 to $11365 \mu \mathrm{g} / \mathrm{L}$. The main characteristic of both observed groups are presented in Table 2. Miners showed some neurotoxic and nephrotoxic sequels of micromercurialism (Kobal et al., 2004; Kobal-Grum et al., 2004). A weak correlation $(r=0.36, p<0.01)$ between systolic blood pressure and average past exposure U-Hg level was found.

The current blood and urine $\mathrm{Hg}$ concentrations were practically on the same level in miners and controls (Table 3). The mean concentration of B-MEL in miners (44.3 ng/L) was significantly higher $(p<0.01)$ than in the controls $(14.9 \mathrm{ng} / \mathrm{L})$. The mean value of U-MEL sulphate $(31.8 \mu \mathrm{g} / \mathrm{L})$ in miners was significantly lower $(p<0.01)$ than in the control group $(46.9 \mu \mathrm{g} / \mathrm{L})$. The mean P-Se in miners $(71.4 \mu \mathrm{g} / \mathrm{L})$ was significantly lower $(p<0.05)$ than in the controls $(77.3 \mu \mathrm{g} / \mathrm{L})$. Among antioxidative enzyme activities (data not presented), only catalase in erythrocytes was significantly higher $(p<0.01)$ in miners $(3.14 \mathrm{MU} / \mathrm{g} \mathrm{Hb})$ than in the controls $(2.65 \mathrm{MU} / \mathrm{g} \mathrm{Hb})$. Among the observed lipid peroxidative products, the mean concentration of U-MDA was statistically higher $(p<0.01)$ in miners $(0.21 \mathrm{mmol} / \mathrm{mmol}$ creatinine $)$ than in the controls $(0.17 \mathrm{mmol} / \mathrm{mmol}$ creatinine).

\subsection{Machine learning results}

The model tree predicting the B-MEL level presented in Table 4 contains a single linear equation comprising group, age, tremor (evaluated with hand-writing test), $\alpha_{1} \mu$ globulin in urine, Se in plasma and cumulative peak U-Hg level. The model represents the miners group. Plasma Se level dominantly, while tremor and cumulative peak U-Hg level partially increased the melatonin concentration in blood. Age dominantly decreased the 
Table 5: Linear regression model (model tree with a single leaf) constructed by M5', describing the concentration of melatonin sulphate in urine (U-MEL sulphate); correlation coefficient $=0.60$. The numbers in brackets correspond to the minimal, maximal, average, median values, and the relative importance factor of each numerical attribute. Relative importance factor is the product of an average value and a corresponding coefficient in the model.

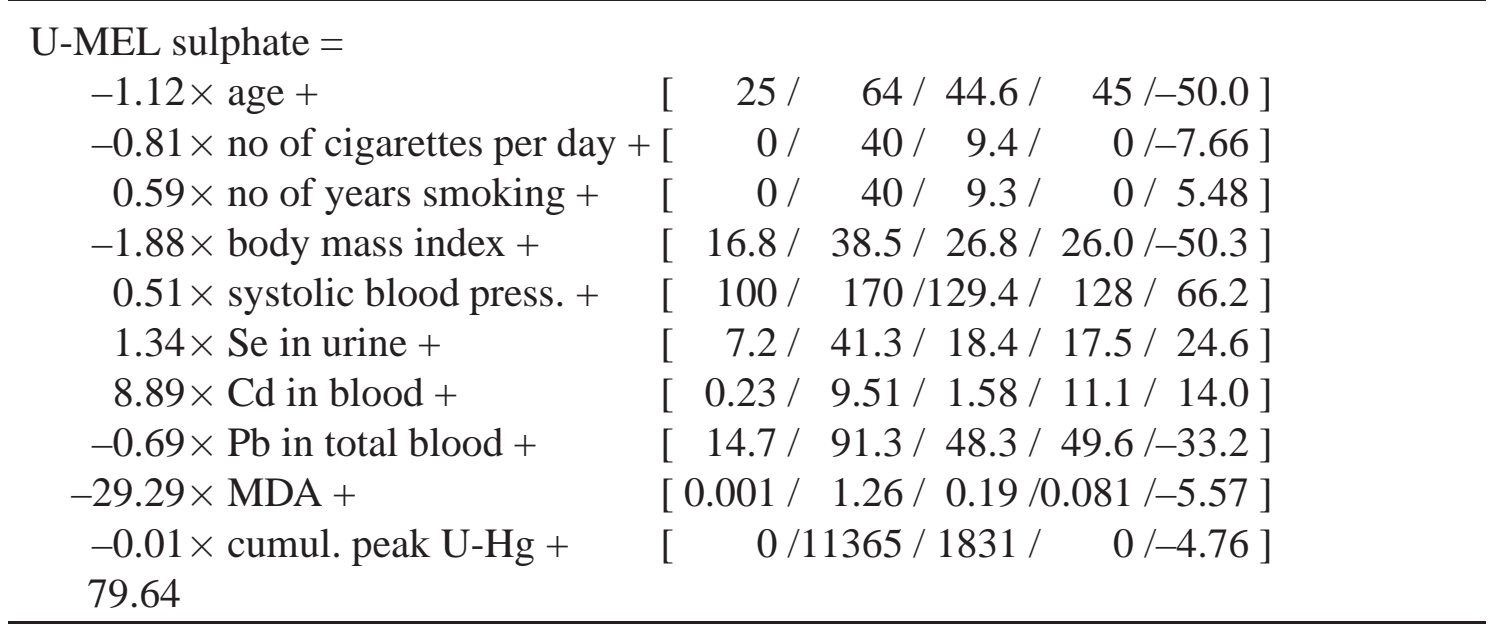

melatonin level in blood. Slight renal tubular disfunction (multicausal etiology) also tends to partially decrease the melatonin concentration in blood.

The model tree predicting the U-MEL concentration is presented in Table 5. It also consists of a single linear equation comprising age, cigarettes consumption, BMI systolic blood pressure, $\mathrm{U}-\mathrm{Se}, \mathrm{Cd}$ and $\mathrm{Pb}$ in blood, U-MDA and cumulative peak $\mathrm{U}-\mathrm{Hg}$ level. Age and $\mathrm{BMI}$ dominantly decreased melatonin sulphate excretion in urine. $\mathrm{Pb}$ level in blood, cigarette consumption per day, cumulative peak U-Hg level and U-MDA also decreased the excretion of melatonin sulphate in urine. Systolic blood pressure and Se concentration in urine were dominantly associated with melatonin sulphate excretion in urine.

\section{Discussion and conclusion}

Melatonin (N-acetyl-5-methoxytryptamine) is a hormone produced mainly in the pineal gland during the dark phase of the circadian cycle. Melatonin metabolises in the liver and is then excreted via urine as melatonin sulphate (U-MEL). The concentration of the metabolite in urine reflects the melatonin concentration in blood (B-MEL) (Reiter et al., 1995). The non-enzymatic reactions of melatonin with free radicals represent a new significant aspect of its biological role (Hardeland et al., 1993). B-MEL concentrations in the present study were significantly higher in miners $(p<0.01)$ in comparison to the controls. U-MEL sulphate tended to be lower in miners which could not be a consequence of lowered melatonin synthesis as B-MEL in miners was very high. To our knowledge such results indicating an increased B-MEL level in $\mathrm{Hg}^{\circ}$ exposed miners have never been previously reported.

The increased level of U-MDA obtained in the present study could represent a moderate increase in lipid peroxidation which could be associated with inorganic $\mathrm{Hg}$ accumulation. Our results are in agreement with the results of some in vitro and in vivo studies 
(Halliwel and Gutteridge, 1989; Lund et al., 1993; Benov et al., 1990) and partly also with the results of (Bulat et al., 1998) where in subjects under ongoing $\mathrm{Hg}^{\circ}$ exposure increased levels of MDA in erythrocytes were found.

In the miners group, Se in plasma together with cumulative peak $\mathrm{U}-\mathrm{Hg}$ are the most important variables which seem to stimulate the melatonin secretion in blood. It also seems that both variables reflect the higher co-accumulation and retention of $\mathrm{Hg}$ and $\mathrm{Se}$ (near equimolar ratio) in central nervous system and endocrine glands (Kosta et al., 1975; Falnoga et al., 2000) that occurred during the past occupational exposure. Age is the most important independent variable which decreases the melatonin synthesis and its concentration in the blood. This effect has also been observed in other studies (Iguchi et al. 1982; Sack et al., 1986). The drop in melatonin synthesis with age is also reflected in the decreased urinary excretion of the chief metabolite melatonin sulphate (Sack et al., 1986).

BMI represents a very important independent variable which reduces the urinary excretion of melatonin sulfate. The influence of BMI on urinary excretion of melatonin sulfate has not been previously elucidated. The increased blood pressure in miners could be hypothetically indirectly associated with higher melatonin sulphate excretion due to the higher melatonin level which acts as an inhibitor of nitric oxide synthase activity and thus on the nitric oxide, a endotetelial-derived relaxing factor, production (Pozo et al., 1994).

Factors that promote the formation of free radicals $(\mathrm{Pb}, \mathrm{Cd}, \mathrm{Hg}$, and tobacco smoking) and affect peroxidative degradation of lipids and phospholipids of cellular membrane additionally decrease the concentration of melatonin in urine. In accordance with this is the negative influence of a lipid peroxidation product (MDA - malondialdehyde) on the melatonin concentration. This supports some assumptions that melatonin also has antioxidative effect. In the process of antioxidative activity melatonin decomposes and we assume this is the reason for decreased urinary excretion of melatonin sulphate in mercury miners in spite of higher melatonin level in blood.

It was surprising to find elevated melatonin synthesis in miners in the period after mercury exposure. Due to the long-term accumulation of mercury in the pineal gland, the opposite was expected. Sleep disturbances typically observed in miners during the state of increased mercury absorption or intoxication (Kobal, 1975a) could be the consequence of mercury interacting with melatonin synthesis in the pineal gland. For the time being, we have no plausible explanation for this highly interesting finding. Increased melatonin secretion could be an adaptive response to free radical production induced by accumulated $\mathrm{Hg}$ (Crawford and Davies, 1994). The mechanism of Hg interaction with the pineal gland is not known.

Machine learning methods enabled us to evaluate interactions between the secretion of melatonin into blood circulation and various indicators of occupational $\mathrm{Hg}$ exposure, some essential metals ( $\mathrm{Se}$ ) and non-occupational exposure to cadmium and lead, as well as some other independent biological variables (age, BMI, and systolic blood pressure). The results of this study are encouraging and point us towards further research of interactions of secretion and excretion of melatonin in subjects occupationally exposed to mercury. 


\section{References}

[1] Aposhian, H.V., Bruce, D.C., Alter, W., Dart, C., Hurlbut, K.M., and Aposhian, M.M. (1992): Urinary mercury after administration of 2,3-dimercaptopropane-1sulfonic acid: correlation with dental amalgam score. Faseb J, 6, 2472-2476.

[2] Benov, L.C., Benchev, I.C., and Monovich, O.H. (1990): Thiol antidotes effect on lipid peroxidation in mercury-poisoned rats. Chem Biol Interact, 76, 321-332.

[3] Breiman, L., Friedman, J.H., Olshen, R.A., and Stone, C.J. (1984): Classification and Regression Trees. Wadsworth.

[4] Bulat, P., Dujić, I., Potkonjak, B., and Vidaković, A. (1998): Activity of glutathione peroxidase and superoxide dismutase in workers occupationally exposed mercury. Int Arch Occup Envirom Health, 71, 537-539.

[5] Crawford, D.R. and Davies, K.J.A. (1994): Adaptive response and oxidative stress. Environ Health Perspect, 102, 25-28.

[6] Falnoga, I., Tušek-Žnidrič, M., Horvat, M., and Stegnar, P. (2000): Mercury, Selenium and Cadmium in human autopsy samples from Idrija residents and mercury mine workers. Environmental Research Section, 84, 211-218.

[7] Halliwell, B. and Gutteridge, J.M.C. (1989): Free Radicals in Biology and Medicine. Oxford: Clarendon Press.

[8] Hardeland, R., Reiter, R.J., Poeggeler, B., and Tan, D.X. (1993): The significance of the metabolism of the neurohormone metatonin: antioxidative protection and formation of bioactive substances. Neuroscience and Biobehavioral Reviews, 17, 347-357.

[9] Iguchi, H., Kato, K.I., and Ibayashi, H. (1982): Age-dependent reduction in serum melatonin concentrations in healthy human subjects. Journal of Clinical Endocrinology and Metabolism, 55, 27-29.

[10] Jansson, G., and Harms-Ringdahl (1993): Stimulating effects in human polymorphonuclear leucocytes. Free Rad Res Commun, 18, 87-98.

[11] Karalič, A. (1992): Employing linear regression in regression tree leaves. In Proceedings of ECAI'92 (European Congress on Artificial Intelligence), Vienna.

[12] Kobal, A.B. (1975): Professional exposure to inorganic mercury and the alterations in serum proteins. In Slovene, M. Sc. Thesis, Zagreb: University of Zagreb.

[13] Kobal, A.B., Horvat, M., Prezelj, M., Sešek-Briški, A., Krsnik, M., Dizdarević, T., Mazej, D., Falnoga, I., Stibilj, V., Arnerić, N., Kobal Grum, D., Osredkar, J. (2004): The impact of long-term past exposure to elemental mercury on antioxidative capacity and lipid peroxidation in mercury miners. J Trace Elem Med Biol, 17, 261-274. 
[14] Kobal-Grum, D., Arnerić, N., Kobal, A.B., Horvat, M., Ženko, B., Džeroski, S., and Osredkar, J. (2004): Can occupational exposure to elementary mercury increase the risk of suicide? Presented at the 7th International Conference on Mercury as a Global Pollutant. RMZ-Mater. Geoenviron., 51, 452-457.

[15] Kosta, L., Byrne, A.R., and Zelenko, V. (1975): Correlation between selenium and mercury in nam following exposure to inorganic mercury. Nature, 254, 238-39.

[16] Leparski, E. and Nüssel, E. (1987): Protocol and Guidelines for Monitoring and Evaluation Procedures, CINDI. Berlin: Springer.

[17] Lund, B.O., Miller, D.M., and Woods, J. S. (1993): Studies on $\mathrm{Hg}$ (II)-induced $\mathrm{H}_{2} \mathrm{O}_{2}$ formation and oxidative stress in vivo and vitro in rat kidney mitohondria. Biochem Pharmacol, 45, 2017-2024.

[18] Pozo, D., Reiter, R.J., Calvo, J.R., and Guerrero, J.M. (1994): Physiological concentrations of melatonin inhibit nitric oxide synthase in rat cerebellum. Life Sci, 55, 455-460.

[19] Quinlan, J.R. (1992): Learning with continuous classes. Proceedings of the Australian Joint Conference on Artificial Intelligence, 343-348, Singapore: World Scientific.

[20] Reiter, R.J., Melchiorri, D., Sewerynek, E., Poeggeler, B., Barlow-Walden, L., Chuang, J., Ortiz, G. G., and Acuna-Castroviejo, D.A. (1995): A review of the evidence supporting metatonin's role as an antioxidant. J Pineal Res, 18, 1-18.

[21] Sack, R.L., Lewy, A.J., Erb, D.L., Vollmer, W.M., and Singer, C.M. (1986): Human melatonin production decreases with age. Journal of Pineal Research, 3, 379-388.

[22] Wang, Y. and Witten, I.H. (1997): Induction of model trees for predicting continuous classes. In Proceedings of the Poster Papers of the European Conference on Machine Learning. Prague: University of Economics, Faculty of Informatics and Statistics.

[23] WHO (1976): Task Group Environmental Health. Environmental Health Criteria 1-Mercury. Geneva: World Health Organization.

[24] WHO (1991): Environmental Health Criteria 118, Inorganic Mercury. Geneva: World Health Organization.

[25] Witten, I.H. and Frank, E. (1999): Data Mining: Practical Machine Learning Tools and Techniques with Java Implementations. San Francisco: Morgan Kaufmann. 